\title{
Improved energy-norm a posteriori error estimates for singularly perturbed reaction-diffusion problems on anisotropic meshes
}

Natalia Kopteva

\begin{abstract}
In the recent article [7] the author obtained residual-type a posteriori error estimates in the energy norm for singularly perturbed semilinear reaction-diffusion equations on unstructured anisotropic triangulations. The error constants in these estimates are independent of the diameters and the aspect ratios of mesh elements and of the small perturbation parameter. The purpose of this note is to improve the weights in the jump residual part of the estimator. This is attained by using a novel sharper version of the scaled trace theorem for anisotropic elements, in which the hat basis functions are involved as weights.
\end{abstract}

\section{Introduction}

Consider finite element approximations to singularly perturbed semilinear reactiondiffusion equations of the form

$$
L u:=-\varepsilon^{2} \triangle u+f(x, y ; u)=0 \quad \text { for }(x, y) \in \Omega, \quad u=0 \quad \text { on } \partial \Omega,
$$

posed in a, possibly non-Lipschitz, polygonal domain $\Omega \subset \mathbb{R}^{2}$. Here $0<\varepsilon \leqslant 1$. We also assume that $f$ is continuous on $\Omega \times \mathbb{R}$ and satisfies $f(\cdot ; s) \in L_{\infty}(\Omega)$ for all $s \in \mathbb{R}$, and the one-sided Lipschitz condition $f(x, y ; v)-f(x, y ; w) \geqslant C_{f}[v-w]$ whenever $v \geqslant w$, with some constant $C_{f} \geqslant 0$. Then there is a unique solution $u \in W_{\ell}^{2}(\Omega) \subseteq$ $W_{q}^{1} \subset C(\bar{\Omega})$ for some $\ell>1$ and $q>2$ [3, Lemma 1]. We additionally assume that $C_{f}+\varepsilon^{2} \geqslant 1$ (as 1.1) can always be reduced to this case by a division by $C_{f}+\varepsilon^{2}$ ).

For this problem, the recent articles [6, 7] gave residual-type a posteriori error estimates on unstructured anisotropic meshes. In particular, in [7] the error was estimated in the energy norm $\|\cdot\| \|_{\varepsilon ; \Omega}$, which is an appropriately scaled $W_{2}^{1}(\Omega)$ norm naturally associated with our problem, defined for any $\mathscr{D} \subseteq \Omega$ by $\|v\|_{\varepsilon ; \mathscr{D}}:=$

Natalia Kopteva

University of Limerick, Limerick, Ireland; e-mail: natalia.kopteva@ul.ie 
$\left\{\varepsilon^{2}\|\nabla v\|_{2 ; \mathscr{D}}^{2}+\|v\|_{2 ; \mathscr{D}}^{2}\right\}^{1 / 2}$. Linear finite elements were used to discretize (1.1) with a piecewise-linear finite element space $S_{h} \subset H_{0}^{1}(\Omega) \cap C(\bar{\Omega})$ relative to a triangulation $\mathscr{T}$, and the the computed solution $u_{h} \in S_{h}$ satisfying

$$
\varepsilon^{2}\left\langle\nabla u_{h}, \nabla v_{h}\right\rangle+\left\langle f_{h}^{I}, v_{h}\right\rangle=0 \quad \forall v_{h} \in S_{h}, \quad f_{h}(\cdot):=f\left(\cdot ; u_{h}\right) .
$$

Here $\langle\cdot, \cdot\rangle$ denotes the $L_{2}(\Omega)$ inner product, and $f_{h}^{I}$ is the standard piecewise-linear Lagrange interpolant of $f_{h}$.

To give a flavour of the results in [7], assuming that all mesh elements are anisotropic, one estimator reduces to

$$
\begin{aligned}
\left\|u_{h}-u\right\|_{\varepsilon ; \Omega} \leqslant & C\left\{\sum_{z \in \mathcal{N}} \min \left\{\left|\omega_{z}\right|, \lambda_{z}\right\}\left\|\varepsilon \llbracket \nabla u_{h} \rrbracket\right\|_{\infty ; \gamma_{z}}^{2}\right. \\
& \left.+\sum_{z \in \mathcal{N}}\left\|\min \left\{1, H_{z} \varepsilon^{-1}\right\} f_{h}^{I}\right\|_{2 ; \omega_{z}}^{2}+\left\|f_{h}-f_{h}^{I}\right\|_{2 ; \Omega}^{2}\right\}^{1 / 2},
\end{aligned}
$$

where $C$ is independent of the diameters and the aspect ratios of elements in $\mathscr{T}$, and of $\varepsilon$. Here $\mathscr{N}$ is the set of nodes in $\mathscr{T}, \llbracket \nabla u_{h} \rrbracket$ is the standard jump in the normal derivative of $u_{h}$ across an element edge, $\omega_{z}$ is the patch of elements surrounding any $z \in \mathscr{N}, \gamma_{z}$ is the set of edges in the interior of $\omega_{z}, H_{z}=\operatorname{diam}\left(\omega_{z}\right)$, and $h_{z} \simeq H_{z}^{-1}\left|\omega_{z}\right|$.

A version of (1.3) obtained in [7] involves a somewhat surprising weight $\lambda_{z}=$ $\varepsilon H_{z}^{2} h_{z}^{-1}$ at the jump residual terms. The main purpose of this note is to improve the jump residual part of the latter estimator and establish its sharper version with a more natural $\lambda_{z}=\varepsilon H_{z}$. This will be attained by employing a novel sharper version of the scaled trace theorem, in which the hat basis functions are involved as weights (see Remark 3.1). As the improvement that we present here applies to the jump residual terms only, we restrict our analysis to these terms.

Note that the new shaper version of the jump residual part of the estimator works not only for (1.3) (see Theorem 4.3 below), but can be also combined with a shaper bound for the interior residual terms given by [7, Theorem 6.2]. The latter is more intricate and was obtained under some additional assumptions on the mesh, so we shall not give it here. Comparing it to (1.3), roughly speaking, the weight $\min \left\{1, H_{z} \varepsilon^{-1}\right\}$ is replaced by a sharper $\min \left\{1, h_{z} \varepsilon^{-1}\right\}$ with a few additional terms included.

Note also that a similar improved jump residual part of the estimator is also obtained in [8, (1.2)] using an entirely different (and more complicated in the context of residual-type estimation) approach for a version of (1.2) (with a special anisotropic quadrature used for the reaction term).

Our interest in locally anisotropic meshes is due to that they offer an efficient way of computing reliable numerical approximations of layer solutions. (In the context of (1.1) with $\varepsilon \ll 1$, see, e.g., [4, 9, 14] and references therein.) But such anisotropic meshes are frequently constructed a priori or by heuristic methods, while the majority of available a posteriori error estimators assume shape regularity of the mesh [1]. In the case of shape-regular triangulations, residual-type a posteriori error estimates for equations of type (1.1) were proved in [16] in the energy norm, and 
more recently in [3] in the maximum norm. The case of anisotropic meshes having a tensor-product structure was addressed in [15] for the Laplace equation and in [5, 2] for problems of type (1.1), with the error estimators given, respectively, in the $H^{1}$ norm and the maximum norm. For unstructured anisotropic meshes, a posteriori error estimates can be found in [10, 12] for the Laplace equation in the $H^{1}$ norm, and in [11, 12] for a linear constant-coefficient version of (1.1) in the energy norm.

Note that the error constants in the estimators of [10,11,12] involve the so-called matching functions; the latter depend on the unknown error and take moderate values only when the grid is either isotropic, or, being anisotropic, is aligned correctly to the solution, while, in general, they may be as large as mesh aspect ratios. The presence of such matching functions in the estimator is clearly undesirable. It is entirely avoided in the more recent papers [6, 7, 8], as well as here.

The paper is organized as follows. In $\sqrt{2}$ and $\sqrt{3}$, we respectively describe our triangulation assumptions and give a novel shaper version of the scaled trace theorem for anisotropic elements. In 84 , we dervie the main result of the paper, a new shaper jump residual part of the estimator. A simplified version of this analysis is given in 84.1 for partially structured anisotropic meshes, while more general anisotropic meshes are addressed in 4.2

Notation. We write $a \simeq b$ when $a \lesssim b$ and $a \gtrsim b$, and $a \lesssim b$ when $a \leqslant C b$ with a generic constant $C$ depending on $\Omega$ and $f$, but not on either $\varepsilon$ or the diameters and the aspect ratios of elements in $\mathscr{T}$. Also, for $\mathscr{D} \subset \bar{\Omega}, 1 \leqslant p \leqslant \infty$, and $k \geqslant 0$, let $\|\cdot\|_{p ; \mathscr{D}}=\|\cdot\|_{L_{p}(\mathscr{D})}$ and $|\cdot|_{k, p ; \mathscr{D}}=|\cdot|_{W_{p}^{k}(\mathscr{D})}$, where $|\cdot|_{W_{p}^{k}(\mathscr{D})}$ is the standard Sobolev seminorm, and osc $(v ; \mathscr{D})=\sup _{\mathscr{D}} v-\inf _{\mathscr{D}} v$ for $v \in L_{\infty}(\mathscr{D})$.

\section{Basic triangulation assumptions}

We shall use $z=\left(x_{z}, y_{z}\right), S$ and $T$ to respectively denote particular mesh nodes, edges and elements, while $\mathscr{N}, \mathscr{S}$ and $\mathscr{T}$ will respectively denote their sets. For each $T \in \mathscr{T}$, let $H_{T}$ be the maximum edge length and $h_{T}:=2 H_{T}^{-1}|T|$ be the minimum height in $T$. For each $z \in \mathscr{N}$, let $\omega_{z}$ be the patch of elements surrounding any $z \in \mathscr{N}$, $\mathscr{S}_{z}$ the set of edges originating at $z$, and

$$
H_{z}:=\operatorname{diam}\left(\omega_{z}\right), \quad h_{z}:=H_{z}^{-1}\left|\omega_{z}\right|, \quad \gamma_{z}:=\mathscr{S}_{z} \backslash \partial \Omega, \quad \dot{\gamma}_{z}:=\left\{S \subset \gamma_{z}:|S| \lesssim h_{z}\right\} .
$$

Throughout the paper we make the following triangulation assumptions.

- Maximum Angle condition. Let the maximum interior angle in any triangle $T \in \mathscr{T}$ be uniformly bounded by some positive $\alpha_{0}<\pi$.

- Local Element Orientation condition. For any $z \in \mathscr{N}$, there is a rectangle $R_{z} \supset \omega_{z}$ such that $\left|R_{z}\right| \simeq\left|\omega_{z}\right|$. Furthermore, if $z \in \mathscr{N} \cap \partial \Omega$ is not a corner of $\Omega$, then $R_{z}$ has a side parallel to the segment $\mathscr{S}_{z} \cap \partial \Omega$.

- Also, let the number of triangles containing any node be uniformly bounded. 
Note that the above conditions are automatically satisfied by shape-regular triangulations.

Additionally, we restrict our analysis to the following two node types defined using a fixed small constant $c_{0}$ (to distinguish between anisotropic and isotropic elements), with the notation $a \ll b$ for $a<c_{0} b$.

(1) Anisotropic Nodes, the set of which is denoted by $\mathscr{N}_{\text {ani }}$, are such that

$$
h_{z} \ll H_{z}, \quad h_{T} \simeq h_{z} \text { and } H_{T} \simeq H_{z} \quad \forall T \subset \omega_{z} .
$$

Note that the above implies that $\mathscr{S}_{z}$ contains at most two edges of length $\lesssim h_{z}$ (see also Fig.3, left).

(2) Regular Nodes, the set of which is denoted by $\mathscr{N}_{\text {reg }}$, are those surrounded by shape-regular mesh elements.

Note that most of our analysis applies to more general node types that were considered in [6, 7]; see Remarks 3.2] and 4.3 for details.

\section{Sharper scaled trace theorem for anisotropic elements}

Our task is to get an improved bound for the jump residual terms (see $I$ in (4.2) below). The key to this will be to employ the following sharper version of the scaled trace theorem for anisotropic elements, which is the main result of this section.

Lemma 3.1. For any node $z \in \mathscr{N}=\mathscr{N}_{\text {ani }} \cup \mathscr{N}_{\text {reg }}$, any function $v \in W_{1}^{1}\left(\omega_{z}\right)$, and any edge $S \subset \gamma_{z}$, one has

$$
\begin{gathered}
\left\|v \phi_{z}\right\|_{1 ; S} \lesssim\|\nabla v\|_{1 ; \omega_{z}}+\|v\|_{1 ; \omega_{z}}\left\{\begin{array}{l}
H_{z}^{-1} \text { if } S \subset \dot{\gamma}_{z}, \\
h_{z}^{-1} \text { if } S \subset \gamma_{z} \backslash \AA_{z},
\end{array}\right. \\
|S|^{-1}\left\|v \phi_{z}\right\|_{1 ; S}^{2} \lesssim\|v\|_{2 ; \omega_{z}}\|\nabla v\|_{2 ; \omega_{z}}+\|v\|_{2 ; \omega_{z}}^{2} \begin{cases}H_{z}^{-1} & \text { if } S \subset \dot{\gamma}_{z}, \\
h_{z}^{-1} & \text { if } S \subset \gamma_{z} \backslash \dot{\gamma}_{z},\end{cases}
\end{gathered}
$$

where $\phi_{z}$ is the hat basis function associated with $z$.

Remark 3.1. Similar versions of the scaled trace theorem for anisotropic elements were obtained in [6, Lemma 3.1] and [7, §3]. Lemma3.1 is an improvement in the sense that in the case of long edges (i.e. $S \subset \gamma_{z} \grave{\gamma}_{z}$ ), the weights at $\|\nabla v\|_{p ; \omega_{z}}$ are sharper. To be more precise, the version of (3.1) in [6, Lemma 3.1] has the weight $H_{z} / h_{z} \gg 1$ at $\|\nabla v\|_{1 ; \omega_{z}}$, while the version of (3.2) given by [7, Corollory 3.2] also involves the weight $H_{z} / h_{z} \gg 1$ at $\|\nabla v\|_{2 ; \omega_{z}}$. Importantly, for the shaper bounds of Lemma 3.1 to hold true, one needs to estimate $\left\|v \phi_{z}\right\|_{p ; S}$ rather than $\|v\|_{p ; S}$ bounded in [6, 7]. Note that this improvement is crucial for getting an improved weight in the jump residual part of our estimator.

Remark 3.2. An inspection of the proof of Lemma 3.1 shows that this lemma remains valid for the more general node types introduced in [7, §2]. 
To prove Lemma 3.1, we shall employ the following auxiliary result.

Lemma 3.2. For any sufficiently smooth function $v \geqslant 0$ on a triangle $T$ with vertices $z, z^{\prime}$ and $z^{\prime \prime}$ and their respective opposite edges $S, S^{\prime}$ and $S^{\prime \prime}$, one has

$$
\begin{aligned}
\sin \angle\left(S^{\prime}, S^{\prime \prime}\right)\left\|v \phi_{z}\right\|_{1 ; S^{\prime}} & \lesssim\|\nabla v\|_{1 ; T}+\left|S^{\prime \prime}\right|^{-1}\|v\|_{1 ; T}, \\
\left|S^{\prime}\right|^{-1}\left\|v \phi_{z}\right\|_{1 ; S^{\prime}} & \lesssim\left|S^{\prime \prime}\right|^{-1}\left\|v \phi_{z}\right\|_{1 ; S^{\prime \prime}}+|S||T|^{-1}\|\nabla v\|_{1 ; T} .
\end{aligned}
$$

Proof. For (3.3a), let $\mu^{\prime \prime}$ be the unit vector along $S^{\prime \prime}$ directed from $z^{\prime}$ to $z$ so that $\nabla \phi_{z} \cdot \mu^{\prime \prime}=\left|S^{\prime \prime}\right|^{-1}$. Note that $\nabla \cdot\left(v \phi_{z} \mu^{\prime \prime}\right)=\nabla\left(v \phi_{z}\right) \cdot \mu^{\prime \prime}$, so the divergence theorem yields

$$
\int_{\partial T}\left(v \phi_{z} \mu^{\prime \prime}\right) \cdot v=\int_{T} \nabla\left(v \phi_{z}\right) \cdot \mu^{\prime \prime}=\int_{T}\left(\phi_{z} \nabla v \cdot \mu^{\prime \prime}+\left|S^{\prime \prime}\right|^{-1} v\right) .
$$

Here, to evaluate the integral $\int_{\partial T}$, note that $\mu^{\prime \prime} \cdot v=0$ on $S^{\prime \prime}$ and $\phi_{z}=0$ on $S$, while $\mu^{\prime \prime} \cdot v=\sin \angle\left(S^{\prime}, S^{\prime \prime}\right)$ on $S^{\prime}$, so $\int_{\partial T}\left(v \phi_{z} \mu^{\prime \prime}\right) \cdot v=\sin \angle\left(S^{\prime}, S^{\prime \prime}\right) \int_{S^{\prime}} v \phi_{z}$. The desired bound $3.3 \mathrm{a}$ follows.

To get (3.3b), we modify the proof of [7, Lemma 7.1]. Set $w=v \phi_{z}$ and also $\mathscr{A}_{S} w:=|S|^{-1} \int_{S} w$ for any edge $S$. Now, with the $\zeta$-axis having the inward normal direction to $S$, and $\hbar:=2|T||S|^{-1}$, one gets $\mathscr{A}_{S^{\prime}} w-\mathscr{A}_{S^{\prime \prime}} w=\hbar^{-1} \int_{0}^{\hbar}\left(\left.w\right|_{S^{\prime}}-\left.w\right|_{S^{\prime \prime}}\right) d \zeta$. This yields (3.3b) as $\phi_{z}$ does not change in the direction normal to $\zeta$.

Proof of Lemma 3.1 First, note that (3.2) follows from (3.1) as $|S|^{-1}\left\|v \phi_{z}\right\|_{1 ; S}^{2} \leqslant$ $\left\|v^{2} \phi_{z}^{2}\right\|_{1 ; S} \leqslant\left\|v^{2} \phi_{z}\right\|_{1 ; S}$, while $\nabla v^{2}=v \nabla v$. With regard to 3.1), it suffices to prove it for the case $v \geqslant 0$, as if $v$ changes sign on $\omega_{z}$, apply (3.1) with $v$ replaced by $v_{\tau}:=\sqrt{v^{2}+\tau^{2}} \geqslant 0$, where $\tau$ is a small positive constant (while $\left|\nabla v_{\tau}\right| \leqslant|\nabla v|$ ), and then let $\tau \rightarrow 0^{+}$so that $v_{\tau} \rightarrow|v|$.

Thus it remains to show (3.1) for $v \geqslant 0$. When $S \subset \dot{\gamma}_{z}$, this bound follows from a similar bound on $\|v\|_{1 ; S}$ in [6, Lemma 3.1]. Now consider $S \subset \gamma_{z} \backslash \dot{\gamma}_{z}$. Then $S$ is a long edge shared by two anisotropic triangles. Consider two cases; see Fig.1. Case (i): If in at least one of these triangles, $T$, the angle at $z$ is $\gtrsim 1$, then an application of (3.3a) yields $\left\|v \phi_{z}\right\|_{1 ; S} \lesssim\|\nabla v\|_{1 ; T}+h_{z}^{-1}\|v\|_{1 ; T}$, and (3.1) follows. Case (ii): Otherwise, in any triangle $T$ sharing the edge $S$, the other edge $S^{\prime \prime}$ originating at $z$ is also of length $\simeq H_{z}$, while the edge opposite to $z$ is of length $\simeq h_{z}$. Then an application of (3.3b) yields $H_{z}^{-1}\left\|v \phi_{z}\right\|_{1 ; S} \lesssim H_{z}^{-1}\left\|v \phi_{z}\right\|_{1 ; S^{\prime \prime}}+H_{z}^{-1}\|\nabla v\|_{1 ; T}$ or, equivalently, $\left\|v \phi_{z}\right\|_{1 ; S} \lesssim\left\|v \phi_{z}\right\|_{1 ; S^{\prime \prime}}+\|\nabla v\|_{1 ; \omega_{z}}$. Thus, a possibly repeated application of (3.3b) reduces this case to case (i); see Fig.1
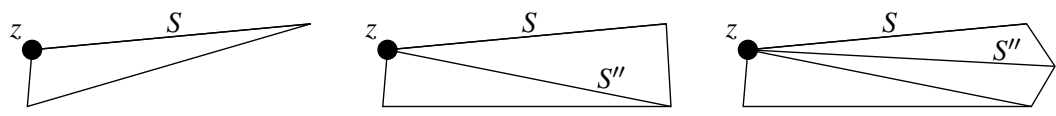

Fig. 1 Illustration to the proof of (3.1) in Lemma 3.1. case (i) (left); case (ii) with a single application of $3.3 \mathrm{~b}$ (centre); case (ii) with a double application of 3.3b) (right). 


\section{A posteriori error bounds for jump residual terms}

Assuming $\left\|u_{h}-u\right\|_{\varepsilon ; \Omega}>0$, let

$$
G:=\frac{u_{h}-u}{\left\|u_{h}-u\right\|_{\varepsilon ; \Omega}} \Rightarrow\|G\|_{\varepsilon ; \Omega}=1, \quad g:=G-G_{h},
$$

where $G_{h} \in S_{h}$ is some interpolant of $G$. Now, a relatively standard calculation yields the following error representation [7, §4]

$$
\begin{aligned}
\left\|u_{h}-u\right\|_{\varepsilon ; \Omega} & \lesssim \sum_{z \in \mathscr{N}} \varepsilon^{2} \int_{\gamma_{z}}\left(g-\bar{g}_{z}\right) \phi_{z} \llbracket \nabla u_{h} \rrbracket \cdot v+\sum_{z \in \mathscr{N}} \int_{\omega_{z}} f_{h}^{I}\left(g-\bar{g}_{z}\right) \phi_{z}+\left|\left\langle f_{h}-f_{h}^{I}, G\right\rangle\right| \\
& =: I+I I+\mathscr{E}_{\text {quad }},
\end{aligned}
$$

which holds for any $G_{h} \in S_{h}$ and any set of real numbers $\left\{\bar{g}_{z}\right\}_{z \in \mathscr{N}}$ such that $\bar{g}_{z}=0$ whenever $z \in \partial \Omega$. (To be precise, $\bar{g}_{z}$ will be specified later as a certain average of $g=G-G_{h}$ near $z$.) Here $\phi_{z}$ denotes the standard hat basis function corresponding to $z \in \mathscr{N}$.

In the following proofs it will be convenient to use, with $p=1,2$, the scaled $W_{p}^{1}(\mathscr{D})$ norm defined by

$$
\|/\|\left\|_{p ; \mathscr{D}}:=\right\| \nabla v\left\|_{p ; \mathscr{D}}+(\operatorname{diam} \mathscr{D})^{-1}\right\| v\left\|_{p ; \mathscr{D}} \Rightarrow\right\|\|v\|\left\|_{p ; \omega_{z}}=\right\| \nabla v\left\|_{p ; \omega_{z}}+H_{z}^{-1}\right\| v \|_{p ; \omega_{z}} .
$$

\subsection{Jump residual for a partially structured anisotropic mesh}

To illustrate our approach in a simpler setting, we first present a version of the analysis for a simpler, partially structured, anisotropic mesh in a square domain $\Omega=(0,1)^{2}$. So, throughout this section, we make the following triangulation assumptions.

A1. Let $\left\{x_{i}\right\}_{i=0}^{n}$ be an arbitrary mesh on the interval $(0,1)$ in the $x$ direction. Then, let each $T \in \mathscr{T}$, for some $i$,

(i) have the shortest edge on the line $x=x_{i}$;

(ii) have a vertex on the line $x=x_{i+1}$ or $x=x_{i-1}$ (see Fig.2, left).

A2. Let $\mathscr{N}=\mathscr{N}_{\text {ani }}$, i.e. each mesh node $z$ satisfies (2.2).

A3. Quasi-non-obtuse anisotropic elements. Let the maximum angle in any triangle be bounded by $\frac{\pi}{2}+\alpha_{1} \frac{h_{T}}{H_{T}}$ for some positive constant $\alpha_{1}$.

These conditions essentially imply that all mesh elements are anisotropic and aligned in the $x$-direction. They also imply that if $x_{z}=x_{i}$, then

$$
\omega_{z} \subseteq \omega_{z}^{*}:=\left(x_{i-1}, x_{i+1}\right) \times\left(y_{z}^{-}, y_{z}^{+}\right), \quad y_{z}^{+}-y_{z}^{-} \simeq h_{z}, \quad \operatorname{diam} \omega_{z}^{*} \simeq H_{z},
$$

where $\left(y_{z}^{-}, y_{z}^{+}\right)$is the range of $y$ within $\omega_{z}$, while $x_{-1}:=x_{0}$ and $x_{n+1}:=x_{n}$. 
Remark 4.1. The above conditions (in particular A3) imply that there is $J \lesssim 1$ such that $\omega_{z}^{*} \subset \omega_{z}^{(J)}$ for all $z \in \mathscr{N}$, where $\omega_{z}^{(0)}:=\omega_{z}$, and $\omega_{z}^{(j+1)}$ denotes the patch of elements in/touching $\omega_{z}^{(j)}$. This conclusion is illustrated on Fig.2](right). (Note that $J=1$ for any non-obtuse triangulation, i.e. for the case $\alpha_{1}=0$ in A3.)

Following [6, 7], the choice of $\bar{g}_{z}$ in (4.2) is related to the orientation of anisotropic elements, and is crucial in our analysis. Let $\bar{g}_{z}=0$ for $z \in \partial \Omega$, and, otherwise, for $x_{z}=x_{i}$ with some $1 \leqslant i \leqslant n-1$, let

$$
\int_{x_{i-1}}^{x_{i+1}}\left(g\left(x, y_{z}\right)-\bar{g}_{z}\right) \varphi_{i}(x) d x=0 .
$$

Here we use the standard one-dimensional hat function $\varphi_{i}(x)$ associated with the mesh $\left\{x_{i}\right\}$ (i.e. it has support on $\left(x_{i-1}, x_{i+1}\right)$, equals 1 at $x=x_{i}$, and is linear on $\left(x_{i-1}, x_{i}\right)$ and $\left.\left(x_{i}, x_{i+1}\right)\right)$.

Theorem 4.1 Let $g=G-G_{h}$ with $G$ from (4.1) and any $G_{h} \in S_{h}$, while

$$
\Theta:=\varepsilon^{2}\|\nabla g\|_{2 ; \Omega}^{2}+\sum_{z \in \mathscr{N}}\left(1+\varepsilon^{2} H_{z}^{-2}\right)\|g\|_{2 ; \omega_{z}}^{2} .
$$

Then $\left\|u_{h}-u\right\|_{\varepsilon ; \Omega} \lesssim I+I I+\mathscr{E}_{\text {quad }}$, where the right-hand side terms are specified in 4.2), and, under conditions $A 1-A 3$,

$$
|I| \lesssim\left\{\Theta \sum_{z \in \mathscr{N}}\left[\min \left\{\left|\omega_{z}\right|, \varepsilon h_{z}\right\}\left(\varepsilon J_{z}^{\circ}\right)^{2}+\min \left\{\left|\omega_{z}\right|, \varepsilon H_{z}\right\}\left(\varepsilon J_{z}\right)^{2}\right]\right\}^{1 / 2}
$$

where $\dot{J}_{z}:=\left\|\llbracket \nabla u_{h} \rrbracket\right\|_{\infty ; \gamma_{z}}$ and $J_{z}:=\left\|\llbracket \nabla u_{h} \rrbracket\right\|_{\infty ; \gamma_{z} \backslash \dot{\gamma}_{z}}$.

Corollary 4.2 Under conditions Al-A3, one has (1.3) with $\lambda_{z}=\varepsilon H_{z}$.

Proof. To get the desired result, combine (4.6) with the bound [7, (5.8)] on II, the straightforward bound $\left|\mathscr{E}_{\text {quad }}\right| \leqslant\left\|f_{h}-f_{h}^{I}\right\|_{2 ; \Omega}$, and $\Theta \lesssim\|G\|_{\mathcal{E} ; \Omega}=1$ (the latter is given by [7, Theorem 7.4] under more general conditions than A1-A3).
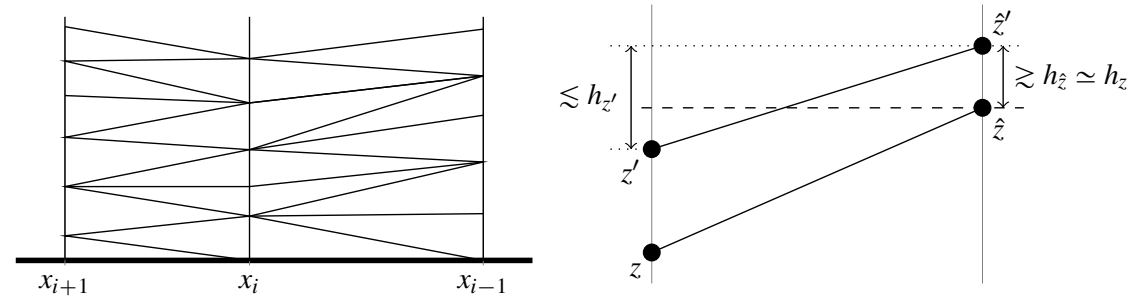

Fig. 2 Partially structured anisotropic mesh (left); illustration for Remark4.1(right): for any fixed edge $z \hat{z}$ and any edge $z^{\prime} \hat{z}^{\prime}$ intercepting the dashed horizontal line via $\hat{z}$, the figure shows that $h_{z} \lesssim h_{z^{\prime}}$, so there is a uniformly bounded number of edges of type $z^{\prime} \hat{z}^{\prime}$, so $\omega_{z}^{*} \subset \omega_{z}^{(J)}$ with $J \lesssim 1$. 
Proof of Theorem 4.1] Split $I$ of (4.2) as $I=\sum_{z \in \mathscr{N}}\left(I_{z}+I_{z}\right)$, where

$$
\check{I}_{z}:=\varepsilon^{2} \int_{\dot{\gamma}_{z}}\left(g-\bar{g}_{z}\right) \phi_{z} \llbracket \nabla u_{h} \rrbracket \cdot v, \quad I_{z}:=\varepsilon^{2} \int_{\gamma_{z} \backslash \gamma_{z}}\left(g-\bar{g}_{z}\right) \phi_{z} \llbracket \nabla u_{h} \rrbracket \cdot v .
$$

First, consider $\bar{g}_{z}$, the definition of which (4.4) implies that $H_{z}\left|\bar{g}_{z}\right| \lesssim\left\|g \varphi_{i}\right\|_{1 ; \bar{S}_{z}}$, where $\bar{S}_{z}$ is the segment joining the points $\left(x_{i-1}, y_{z}\right)$ and $\left(x_{i+1}, y_{z}\right)$, so $\left|\bar{S}_{z}\right| \simeq H_{z}$. Versions of (3.1) and (3.2) then respectively yield

$$
H_{z}\left|\bar{g}_{z}\right| \lesssim\|\nabla g\|_{1 ; \omega_{z}^{*}}+h_{z}^{-1}\|g\|_{1 ; \omega_{z}^{*}}, H_{z}\left|\bar{g}_{z}\right|^{2} \lesssim\|g\|_{2 ; \omega_{z}^{*}}\left(\|\nabla g\|_{2 ; \omega_{z}^{*}}+h_{z}^{-1}\|g\|_{2 ; \omega_{z}^{*}}\right) .
$$

These two bounds will be used when estimating both $\stackrel{I}{z}_{z}$ and $I_{z}$.

We now proceed to estimating $\stackrel{\circ}{z}_{z}$. Note that (3.1) implies that $\left\|\left(g-\bar{g}_{z}\right) \phi_{z}\right\|_{1 ; \gamma_{z}} \lesssim$ $\| / / g / /{ }_{1 ; \omega_{z}^{*}} \lesssim\left|\omega_{z}\right|^{1 / 2} / / \mid / / / 2 ; \omega_{z}^{*}$, where we also used $\left\|\bar{g}_{z} \phi_{z}\right\|_{1 ; \gamma_{z}} \simeq h_{z}\left|\bar{g}_{z}\right|$ combined with the first bound from (4.8). Similarly, $\left\|\left(g-\bar{g}_{z}\right) \phi_{z}\right\|_{1 ; \gamma_{z}}^{2} \lesssim h_{z}\|g\|_{2 ; \omega_{z}^{*}} / / g / \| 2_{2 ; \omega_{z}^{*}}$, where we employed (3.2) and the second bound from (4.8). Now, from the definition of $I_{z}$ in (4.7) combined with the two bounds on $\left\|\left(g-\bar{g}_{z}\right) \phi_{z}\right\|_{1 ; \gamma_{z}}$, one concludes that

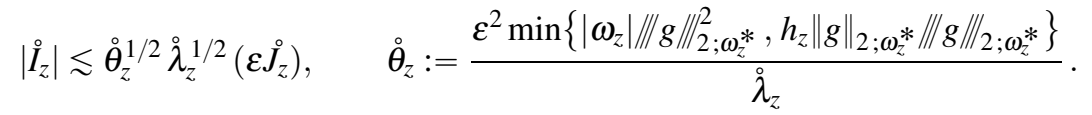

Set $\AA_{z}:=\min \left\{\left|\omega_{z}\right|, \varepsilon h_{z}\right\}$. Then, to get the bound of type (4.6) for $\sum_{z \in \mathscr{N}} \check{I}_{z}$, it remains to show that $\sum_{z \in \mathscr{N}} \stackrel{\circ}{z}_{z} \lesssim \Theta$. For the latter, in view of

$$
\min \left\{a a^{\prime}, b b^{\prime}\right\} / \min \left\{a^{\prime}, b^{\prime}\right\} \leqslant a+b \quad \forall a, a^{\prime}, b, b^{\prime}>0,
$$

one gets $\stackrel{\circ}{z}_{z} \lesssim \varepsilon^{2} / / g / / \mathbb{2}_{2 ; \omega_{z}^{*}}^{2}+\varepsilon\|g\|_{2 ; \omega_{z}^{*}} / / g / / 2_{2 ; \omega_{z}^{*}}$, which leads to $\sum_{z \in \mathscr{N}} \stackrel{\circ}{z}_{z} \lesssim \Theta$, also using Remark 4.1

For $I_{z}$, first, recall the bound $\left|I_{z}\right| \lesssim \varepsilon / / / / / \|_{1 ; \omega_{z}^{*}}\left(\varepsilon J_{z}\right)$ from [7, (5.12)], which implies $\left|I_{z}\right| \lesssim \varepsilon\left|\omega_{z}\right|^{1 / 2} / / / g / / 2 ; \omega_{z}^{*}\left(\varepsilon J_{z}\right)$. An alternative bound on $I_{z}$ follows from $\left\|\left(g-\bar{g}_{z}\right) \phi_{z}\right\|_{1 ; \gamma_{z} \dot{\gamma}_{z}}^{2} \lesssim H_{z}\|g\|_{2 ; \omega_{z}^{*}}\left(\|\nabla g\|_{2 ; \omega_{z}^{*}}+h_{z}^{-1}\|g\|_{2 ; \omega_{z}^{*}}\right)$, where the latter is obtained by an application of (3.2) for $g$, while the second bound from (4.8) is employed for $\bar{g}_{z}$. Combining the two bounds on $I_{z}$, we arrive at

$$
\begin{aligned}
\left|I_{z}\right| & \lesssim \theta_{z}^{1 / 2} \lambda_{z}^{1 / 2}\left(\varepsilon J_{z}\right), \\
\theta_{z} & :=\frac{\varepsilon^{2} \min \left\{\left|\omega_{z}\right| / / / g\left\|/ \int_{2 ; \omega_{z}^{*}}^{2}, H_{z}\right\| g \|_{2 ; \omega_{z}^{*}}\left(\|\nabla g\|_{2 ; \omega_{z}^{*}}+h_{z}^{-1}\|g\|_{2 ; \omega_{z}^{*}}\right)\right\}}{\lambda_{z}} .
\end{aligned}
$$

Here set $\lambda_{z}:=\min \left\{\left|\omega_{z}\right|, \varepsilon H_{z}\left(1+\varepsilon h_{z}^{-1}\right)\right\}$. Now, again using (4.9), one gets

$$
\theta_{z} \lesssim \varepsilon^{2} / / g\|\|_{2 ; \omega_{z}^{*}}^{2}+\varepsilon\|g\|_{2 ; \omega_{z}^{*}}\left(\|\nabla g\|_{2 ; \omega_{z}^{*}}+h_{z}^{-1}\|g\|_{2 ; \omega_{z}^{*}}\right) /\left(1+\varepsilon h_{z}^{-1}\right),
$$

and hence $\sum_{z \in \mathscr{N}} \theta_{z} \lesssim \Theta$. Finally, to get the bound of type (4.6) for $\sum_{z \in \mathscr{N}} I_{z}$, it remains to note that $\lambda_{z}=\min \left\{\left|\omega_{z}\right|, \varepsilon H_{z}\left[1+\varepsilon h_{z}^{-1}\right]\right\} \simeq \min \left\{\left|\omega_{z}\right|, \varepsilon H_{z}\right\}$. 
Remark 4.2. While the definition (4.4) for $\bar{g}_{z}$ is quite different from a standard choice (see, e.g., [13, Lecture 5]), its role may not be immediately obvious in the proof of Theorem 4.1 To clarify this, note that it is crucial for the bound $\left|I_{z}\right| \lesssim \varepsilon / / / / / 1_{1 ; \omega_{*}^{*}}\left(\varepsilon J_{z}\right)$ quoted from [7, (5.12)]. To be more precise, the latter bound is obtained in [7] using the representation

$$
\begin{aligned}
I_{z}=I_{z}^{\prime}+I_{z}^{\prime \prime}+I_{z}^{\prime \prime \prime} & :=\varepsilon^{2} \int_{\gamma_{z} \backslash \dot{\gamma}_{z}}\left(g-\bar{g}_{z}\right) \phi_{z} \llbracket \partial_{x} u_{h} \rrbracket v_{x} \\
& +\varepsilon^{2} \int_{\gamma_{z} \backslash \dot{\gamma}_{z}}\left[g-g\left(x, y_{z}\right)\right] \phi_{z} \llbracket \partial_{y} u_{h} \rrbracket v_{y} \\
& +\varepsilon^{2} \int_{\gamma_{z} \backslash \dot{\gamma}_{z}}\left[g\left(x, y_{z}\right)-\bar{g}_{z}\right] \phi_{z} \llbracket \partial_{y} u_{h} \rrbracket v_{y},
\end{aligned}
$$

where $\llbracket w \rrbracket$, for any $w$, is understood as the jump in $w$ across any edge in $\gamma_{z}$ evaluated in the anticlockwise direction about $z$. Importantly, here $I_{z}^{\prime \prime \prime}=0$ due to our choice of $\bar{g}_{z}$ (as well as due to the partial structure of our mesh; in a more general case, the estimation of $I_{z}^{\prime \prime \prime}$ is more intricate).

\subsection{Jump residual for for general anisotropic meshes}

Theorem 4.3 Suppose that $\mathscr{N}=\mathscr{N}_{\text {ani }} \cup \mathscr{N}_{\text {reg }}$ and all corners of $\Omega$ are in $\mathscr{N}_{\text {reg. }}$ Let $g=G-G_{h}$ with $G$ from (4.1) and any $G_{h} \in S_{h}$, while $\Theta$ is defined by (4.5). Then $\left\|u_{h}-u\right\|_{\varepsilon ; \Omega} \lesssim I+I I+\mathscr{E}_{\text {quad, }}$ where the right-hand side terms are specified in (4.2), and

$$
|I| \lesssim\left\{\Theta \sum_{z \in \mathscr{N}} \min \left\{\left|\omega_{z}\right|, \varepsilon H_{z}\right\}\left\|\varepsilon \llbracket \nabla u_{h} \rrbracket\right\|_{\infty ; \gamma_{z}}^{2}\right\}^{1 / 2}
$$

Corollary 4.4 Under the conditions of Theorem 4.3 one has (1.3) with $\lambda_{z}=\varepsilon H_{z}$.

Proof. To get the desired result, combine (4.12) with the bound [7, (6.2)] on II, the straightforward bound $\left|\mathscr{E}_{\text {quad }}\right| \leqslant\left\|f_{h}-f_{h}^{I}\right\|_{2 ; \Omega}$, and $\Theta \lesssim\|G\|_{\varepsilon ; \Omega}=1$ (the latter follows from [7, Theorem 7.4] as $\mathscr{N}=\mathscr{N}_{\text {ani }} \cup \mathscr{N}_{\text {reg }}$ ).

Remark 4.3. In view of Remark 3.2 an inspection of the proof of Theorem 4.3 shows that this theorem remains valid for the more general node types introduced in [7, §2], and furthermore, can be combined with a shaper bound for the interior residual terms given by [7, Theorem 6.2].

Proof of Theorem 4.3. Split $I$ of (4.2) as $I=\sum_{z \in \mathscr{N}} I_{z}$, where $I_{z}$ is defined as in (4.7), only with $\gamma_{z} \backslash \dot{\gamma}_{z}$ replaced by $\gamma_{z}$. It suffices to show that for some edge subset $\mathscr{S}^{*} \subset \mathscr{S}$ with some quantities $\mathscr{I}_{S ; z}$ associated with any $S \in \mathscr{S}_{z} \cap \mathscr{S}^{*}$ (to be specified below), one has 


$$
\begin{aligned}
\sum_{z \in \mathscr{N}} \sum_{S \in \mathscr{S}_{z} \cap \mathscr{S}^{*}} \mathscr{I}_{S ; z} & =0 \\
\left|I_{z}+\sum_{S \in \mathscr{S}_{z} \cap \mathscr{S}^{*}} \mathscr{I}_{S ; z}\right| & \lesssim \varepsilon /\|g\|\left\|1 ; \omega_{z}\right\| \varepsilon \llbracket \nabla u_{h} \rrbracket \|_{\infty ; \gamma_{z}} \\
& \lesssim \varepsilon\left|\omega_{z}\right|^{1 / 2}\|/\| g\left\|/ 2_{2 ; \omega_{z}}\right\| \varepsilon \llbracket \nabla u_{h} \rrbracket \|_{\infty ; \gamma_{z}}, \\
\left|I_{z}\right|+\sum_{S \in \mathscr{S}_{z} \cap \mathscr{S}^{*}}\left|\mathscr{I}_{S ; z}\right| & \lesssim \varepsilon\left\{H_{z}\|g\|_{2 ; \omega_{z}}\left(\|\nabla g\|_{2 ; \omega_{z}}+h_{z}^{-1}\|g\|_{2 ; \omega_{z}}\right)\right\}^{1 / 2}\left\|\varepsilon \llbracket \nabla u_{h} \rrbracket\right\|_{\infty ; \gamma_{z}}
\end{aligned}
$$

Indeed, 4.13a) implies that $I=\sum_{z \in \mathscr{N}} I_{z}=\sum_{z \in \mathscr{N}}\left(I_{z}+\sum_{S \in \mathscr{S}_{z} \cap \mathscr{S} *} \mathscr{I}_{S ; z}\right)$, while 4.13b), 4.13c yield

$$
\begin{aligned}
& \left|I_{z}+\sum_{S \in \mathscr{S}_{z} \cap \mathscr{S}^{*}} \mathscr{I}_{S ; z}\right| \lesssim \theta_{z}^{1 / 2} \lambda_{z}^{1 / 2}\left\|\varepsilon \llbracket \nabla u_{h} \rrbracket\right\|_{\infty ; \gamma_{z}}, \\
& \theta_{z}:=\frac{\varepsilon^{2} \min \left\{\left.\left|\omega_{z}\right|\|/ /\|\right|_{2 ; \omega_{z}} ^{2}, H_{z}\|g\|_{2 ; \omega_{z}}\left(\|\nabla g\|_{2 ; \omega_{z}}+h_{z}^{-1}\|g\|_{2 ; \omega_{z}}\right)\right\}}{\lambda_{z}} .
\end{aligned}
$$

Here set $\lambda_{z}:=\min \left\{\left|\omega_{z}\right|, \varepsilon H_{z}\left(1+\varepsilon h_{z}^{-1}\right)\right\}$. Then (4.14) becomes a version of (4.10) with $\omega_{z}^{*}$ replaced by $\omega_{z}$, so proceeding as in the proof of Theorem 4.1 (i.e. again employing (4.9)), one gets a version (4.11) with $\omega_{z}^{*}$ replaced by $\omega_{z}$, which leads to $\sum_{z \in \mathscr{N}} \theta_{z} \lesssim \Theta$. Now, to get the desired bound (4.12), it remains to note that $\lambda_{z}=$ $\min \left\{\left|\omega_{z}\right|, \varepsilon H_{z}\left(1+\varepsilon h_{z}^{-1}\right)\right\} \simeq \min \left\{\left|\omega_{z}\right|, \varepsilon H_{z}\right\}$.

So, to complete the proof, we need to establish (4.13). Relations (4.13a) and (4.13b) immediately follow from [8, (6.10), (6.11a), (6.11b)] for a certain choice of $\left\{\bar{g}_{z}\right\}_{z \in \mathscr{N}}$, the edge subset $\mathscr{S}^{*} \subset \mathscr{S}$ and the quantities $\mathscr{I}_{S ; z}$ associated with any $S \in \mathscr{S}_{z} \cap \mathscr{S}^{*}$. We need to recall their definitions to prove the remaining required bound $(4.13 \mathrm{c})$ (which is a sharper version of [8, ((6.11c)]).

First, we recall the definition of $\left\{\bar{g}_{z}\right\}_{z \in \mathscr{N}}$. In view of the Local Element Orientation condition (see $\S 2$ ), for each fixed $z \in \mathscr{N}$, introduce the following local notation. Let the local cartesian coordinates $(\xi, \eta)$ be such that $z=(0,0)$, and the unit vector $\mathrm{i}_{\xi}$ in the $\xi$ direction lies along the longest edge $\hat{S}_{z} \in \mathscr{S}_{z}$ (see Fig. 3 (left)). For $z \in \mathscr{N}_{\text {ani }} \cap \partial \Omega$ (hence $z$ is not a corner of $\Omega$ ), let $i_{\xi}$ be either parallel or orthogonal to $\partial \Omega$ at $z$ (depending on whether $\omega_{z}$ is, roughly speaking, parallel or orthogonal to $\partial \Omega)$.

Next, split $\mathscr{S}_{z}=\dot{\mathscr{S}}_{z} \cup \mathscr{S}_{z}^{+} \cup \mathscr{S}_{z}^{-}$, where $\dot{\mathscr{S}}_{z}=\left\{S \subset \mathscr{S}_{z}:|S| \lesssim h_{z}\right\}$ (so $\dot{\gamma}_{z}=$ $\left.\dot{\mathscr{S}}_{z} \backslash \partial \Omega\right)$. Here we also use $\mathscr{S}_{z}^{ \pm}:=\left\{S \subset \mathscr{S}_{z} \backslash \dot{\mathscr{S}}_{z}: S_{\xi} \subset \mathbb{R}_{ \pm}\right\}$, where $S_{\xi}=\operatorname{proj}_{\xi}(S)$ denotes the projection of $S$ onto the $\xi$-axis. Now, let $\left(\xi_{z}^{-}, \xi_{z}^{+}\right)$be the maximal interval such that $\left(\xi_{z}^{-}, 0\right) \subset S_{\xi}$ for all $S \in \mathscr{S}_{z}^{-}$and $\left(0, \xi_{z}^{+}\right) \subset S_{\xi}$ for all $S \in \mathscr{S}_{z}^{+}$. Also, let $\varphi_{z}(\xi)$ be the standard piecewise-linear hat-function with support on $\left(\xi_{z}^{-}, \xi_{z}^{+}\right)$and equal to 1 at $\xi=0$. Note that if $\mathscr{S}_{z}^{-}=\varnothing\left(\right.$ and $\left.\mathscr{S}_{z}^{+}=\varnothing\right)$, then we set $\xi_{z}^{-}=0$ (and $\xi_{z}^{+}=0$ ) and do not use $\varphi_{z}$ for $\xi<0$ (and $\xi>0$ ). 
Next, for $\xi \in\left[\xi_{z}^{-}, \xi_{z}^{+}\right]$define a continuous function $\bar{\eta}_{z}(\xi)$ as follows: (i) $\bar{\eta}_{z}(\xi)$ is linear on $\left[\xi_{z}^{-}, 0\right]$ and $\left[0, \xi_{z}^{+}\right]$; (ii) $\bar{\eta}_{z}(0)=0$; (iii) $\left(\xi, \bar{\eta}_{z}(\xi)\right) \in \omega_{z}$ for all $\xi \in\left(\xi_{z}^{-}, \xi_{z}^{+}\right)$. (For example, one may choose $\bar{\eta}_{z}(\xi)$ so that $\left\{\left(\xi, \bar{\eta}_{z}(\xi)\right): \xi \in\left(\xi_{z}^{-}, 0\right)\right\}$ lies on any edge in $\mathscr{S}_{z}^{-}$, while $\left\{\left(\xi, \bar{\eta}_{z}(\xi)\right): \xi \in\left(0, \xi_{z}^{+}\right)\right\}$lies on any edge in $\mathscr{S}_{z}^{+}$; see Fig. 3 (left).)

We are now prepared to specify $\bar{g}_{z}$. Let $\bar{g}_{z}:=0$ if $z \in \partial \Omega$ or $z \in \mathscr{N}_{\text {reg }}$ (as for the latter, $\xi_{z}^{-}=\xi_{z}^{+}=0$ ), and, otherwise, let

$$
\int_{\xi_{z}^{-}}^{\xi_{z}^{+}}\left[g\left(\xi, \bar{\eta}_{z}(\xi)\right)-\bar{g}_{z}\right] \varphi_{z}(\xi) d \xi=0 .
$$

Also, let $\bar{S}_{z}^{-}:=\left\{\left(\xi, \bar{\eta}_{z}(\xi)\right): \xi \in\left(\xi_{z}^{-}, 0\right)\right\}$ and $\bar{S}_{z}^{+}:=\left\{\left(\xi, \bar{\eta}_{z}(\xi)\right): \xi \in\left(0, \xi_{z}^{+}\right)\right\}$, i.e. $\bar{S}_{z}^{ \pm}$is the segment joining $(0,0)$ and $\left(\xi_{z}^{ \pm}, \bar{\eta}_{z}\left(\xi_{z}^{ \pm}\right)\right)$.

We can now proceed to getting a bound of type (4.13c for $\left|I_{z}\right|$. First, con-

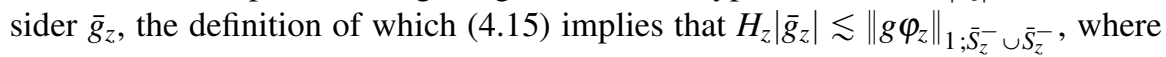
$\left|S_{z}^{-} \cup \bar{S}_{z}^{-}\right| \simeq H_{z}$. Using (3.1) and (3.2) then yields a version of (4.8), only with $\omega_{z}^{*}$ replaced by $\omega_{z}$ (as now $\bar{S}_{z}^{-} \cup \bar{S}_{z}^{+} \subset \omega_{z}$ ). Next, we get $\left\|\left(g-\bar{g}_{z}\right) \phi_{z}\right\|_{1 ; \gamma_{z}}^{2} \lesssim$ $H_{z}\|g\|_{2 ; \omega_{z}}\left(\|\nabla g\|_{2 ; \omega_{z}}+h_{z}^{-1}\|g\|_{2 ; \omega_{z}}\right)$, which is obtained by an application of (3.2) for $g$, while the second bound from (4.8) is employed for $\bar{g}_{z}$. Combining this with the definition of $I_{z}$ immediately yields a bound of type (4.13c $)$ for $\left|I_{z}\right|$.

To establish a bound of type (4.13c) for $\left|\mathscr{I}_{S ; z}\right|$, we now recall the definitions of the edge subset $\mathscr{S}^{*} \subset \mathscr{S}$ and the quantities $\mathscr{I}_{S ; z}$ associated with any $S \in \mathscr{S}_{z} \cap \mathscr{S}^{*}$ from [7]. Let $\mathscr{S}^{*}:=\cup_{z \in \mathscr{N}_{\text {ani }} \mid \partial \Omega} \mathscr{S}_{z}^{\circ}$, and for any $S \in \mathscr{S}^{*}$ with endpoints $z$ and $z^{\prime}$, define

$$
\mathscr{I}_{S ; z}:=\varepsilon^{2} \alpha_{S} \mu_{z z^{\prime}} \cdot \mathrm{i}_{\xi *} J_{S}, \quad \alpha_{S}:=\int_{0}^{\xi_{S}^{+}}\left[g\left(\xi^{*}, \bar{\eta}_{S}\left(\xi^{*}\right)\right)-\bar{g}_{S}\right] \varphi_{S}\left(\xi^{*}\right) d \xi^{*} .
$$

Here $J_{S}$ is the standard signed version of $\left|\llbracket \nabla u_{h} \rrbracket\right|$ on $S, \mu_{z z^{\prime}}$ is the unit vector directed from $z$ to $z^{\prime}$, and $\mathrm{i}_{\xi *}$ is the unit vector along the $\xi^{*}$-axis. The local cartesian coordinates $\left(\xi^{*}, \eta^{*}\right)$ are associated with $S$ and coincide with the local coordinates $(\xi, \eta)$ associated with either $z \in \mathscr{N}_{\text {ani }} \backslash \partial \Omega$ or $z^{\prime} \in \mathscr{N}_{\text {ani }} \backslash \partial \Omega$ (at least one of them is always in $\mathscr{N}_{\text {ani }} \backslash \partial \Omega$ ). The above $\alpha_{S}$ is defined by a version
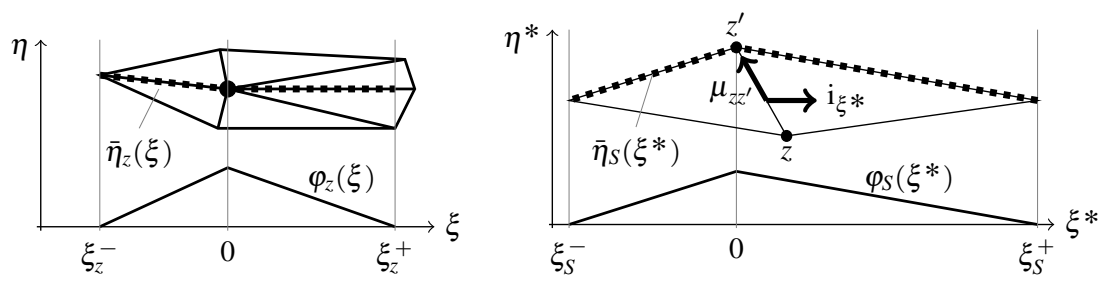

Fig. 3 Local notation associated with a node $z \in \mathscr{N}_{\text {ani }}$ (left), and an edge $S \in \mathscr{S}^{*}$ with endpoints $z$ and $z^{\prime}$ (right). 
$\int_{\xi_{S}^{-}}^{\xi_{S}^{+}}\left[g\left(\xi^{*}, \bar{\eta}_{S}\left(\xi^{*}\right)\right)-\bar{g}_{S}\right] \varphi_{S}\left(\xi^{*}\right) d \xi^{*}=0$ of 4.15). The one-dimensional hat function $\varphi_{S}\left(\xi^{*}\right)$ is associated with the interval $\left(\xi_{S}^{-}, \xi_{S}^{+}\right)$; the latter is the projection of $\omega_{z} \cap \omega_{z^{\prime}}$ (which includes at most two triangles) onto the $\xi^{*}$-axis. The piecewiselinear function $\bar{\eta}_{S}\left(\xi^{*}\right)$ is defined similarly to $\bar{\eta}_{z}(\xi)$ under the restriction that any point $\left(\xi^{*}, \bar{\eta}\left(\xi^{*}\right)\right) \in \omega_{z} \cap \omega_{z^{\prime}}$ (see Fig. 3) right)).

Under this definition, a bound of type (4.13c) for $\left|\mathscr{I}_{S ; z}\right|$ is established similarly to a similar bound for $\left|I_{z}\right|$. (Note also that $\mu_{z z^{\prime}}+\mu_{z^{\prime} z}=0$ in (4.16), so $\mathscr{I}_{S ; z}+\mathscr{I}_{S ; z^{\prime}}=0$, which implies (4.13a).) This completes the proof of (4.13), and hence of (4.12).

\section{References}

1. Ainsworth, M., Oden, J. T.: A posteriori error estimation in finite element analysis. WileyInterscience, New York (2000)

2. Chadha, N. M., Kopteva, N.: Maximum norm a posteriori error estimate for a $3 \mathrm{~d}$ singularly perturbed semilinear reaction-diffusion problem. Adv. Comput. Math. 35, 33-55 (2011)

3. Demlow, A., Kopteva, N.: Maximum-norm a posteriori error estimates for singularly perturbed elliptic reaction-diffusion problems. Numer. Math. 133, 707-742 (2016)

4. Kopteva, N.: Maximum norm error analysis of a $2 \mathrm{~d}$ singularly perturbed semilinear reactiondiffusion problem. Math. Comp. 76, 631-646 (2007)

5. Kopteva, N.: Maximum norm a posteriori error estimate for a $2 \mathrm{~d}$ singularly perturbed reactiondiffusion problem. SIAM J. Numer. Anal. 46, 1602-1618 (2008)

6. Kopteva, N.: Maximum-norm a posteriori error estimates for singularly perturbed reactiondiffusion problems on anisotropic meshes. SIAM J. Numer. Anal. 53, 2519-2544 (2015)

7. Kopteva, N.: Energy-norm a posteriori error estimates for singularly perturbed reactiondiffusion problems on anisotropic meshes. Numer. Math., 137, 607-642 (2017)

8. Kopteva, N.: Fully computable a posteriori error estimator using anisotropic flux equilibration on anisotropic meshes. Submitted for publication (2017), arXiv:1704.04404

9. Kopteva, N., O'Riordan, E.: Shishkin meshes in the numerical solution of singularly perturbed differential equations. Int. J. Numer. Anal. Model. 7, 393-415 (2010)

10. Kunert, G.: An a posteriori residual error estimator for the finite element method on anisotropic tetrahedral meshes. Numer. Math. 86, 471-490 (2000)

11. Kunert, G.: Robust a posteriori error estimation for a singularly perturbed reaction-diffusion equation on anisotropic tetrahedral meshes. Adv. Comput. Math. 15, 237-259 (2001)

12. Kunert, G., Verfürth, R.: Edge residuals dominate a posteriori error estimates for linear finite element methods on anisotropic triangular and tetrahedral meshes. Numer. Math. 86, 283-303 (2000)

13. Nochetto, R.H.: Pointwise a posteriori error estimates for monotone semi-linear equations. Lecture Notes at 2006 CNA Summer School Probabilistic and Analytical Perspectives on Contemporary PDEs, http://www.math.cmu.edu/cna/Summer06/lecturenotes/nochetto/

14. Roos, H.-G., Stynes, M., Tobiska, T.: Robust Numerical Methods for Singularly Perturbed Differential Equations. Springer, Berlin (2008)

15. Siebert, K. G.: An a posteriori error estimator for anisotropic refinement. Numer. Math. 73, 373-398 (1996)

16. Verfürth, R.: Robust a posteriori error estimators for a singularly perturbed reaction-diffusion equation. Numer. Math. 78, 479-493 (1998) 